\title{
Chapter 15.
}

\section{Conjugated polymers: Relationship between morphology and optical properties}

Maria Isabel Alonso and Mariano Campoy-Quiles

Institut de Ciència de Materials de Barcelona (ICMAB-CSIC), Campus UAB, 08193 Bellaterra, Spain.

\begin{abstract}
In this Chapter we will start by briefly summarising the basic concepts of the electronic structure of conjugated polymers. This will enable the discussion of the relevant descriptions of the dielectric function. We will relate these descriptions to the model parameterisations which are used in advanced ellipsometric analysis of thin films such as those used in devices for organic photovoltaics (OPVs) and light emitting diodes (OLEDs). Amongst other things, such parametric descriptions are useful to deal with structural changes in conjugated polymer thin films. Once the models are presented, we will provide representative examples of the nexus between morphology and optical constants, and how the latter can be employed to infer aspects of the former. First, we will discuss how chain conformation affects the optical properties. Then, we will explain the anisotropic behaviour of conjugated polymer films due to their intrinsic molecular anisotropy and review different cases (f. i., oriented films or semicrystalline polymers). We will also describe structural changes that occur upon blending polymers with fullerenes and concomitant variations of the optical properties. Here we will focus on state of the art low band gap polymers mixed with fullerenes. Finally, real-time ellipsometric experiments in which these structure-property relationships can be exploited will be presented.
\end{abstract}

\subsection{Introduction}

Conjugated polymers are technologically interesting materials sharing the general advantages of polymers and the optical and electrical properties characteristic of semiconductors.[1] This unique combination of features offers substantial benefits for applications in the fields of photovoltaics, [2] light emitting diodes, [3] and transistors, [4] where different molecular structures, morphologies and device architectures provide the 
required useful functionalities (cf. Chap. 12). Ideally, as macromolecules, polymers can be designed and synthesised to achieve the desired behaviour and can be processed using low-cost solution methods to engineer a wealth of devices. Although solution processing is probably the greatest benefit for polymer electronics, many properties of the polymer electronic devices depend entirely on the processing conditions and with them, their device performance.[5] Some technologically relevant donor polymers and the ubiquitous acceptor fullerene derivative PCBM (Phenyl-C60-butyric acid methyl ester) are depicted in Fig. 15.1. Spectroscopic ellipsometry has emerged as a useful technique for the design of new materials, control of microstructure and characterization and optimization of device-layer films.[6] It helps to evaluate not only structural features such as film thicknesses and composition profiles but it is also the technique of choice to study the basic optical properties of the active organic semiconducting films, which result from the combination of both chemical nature and morphology.

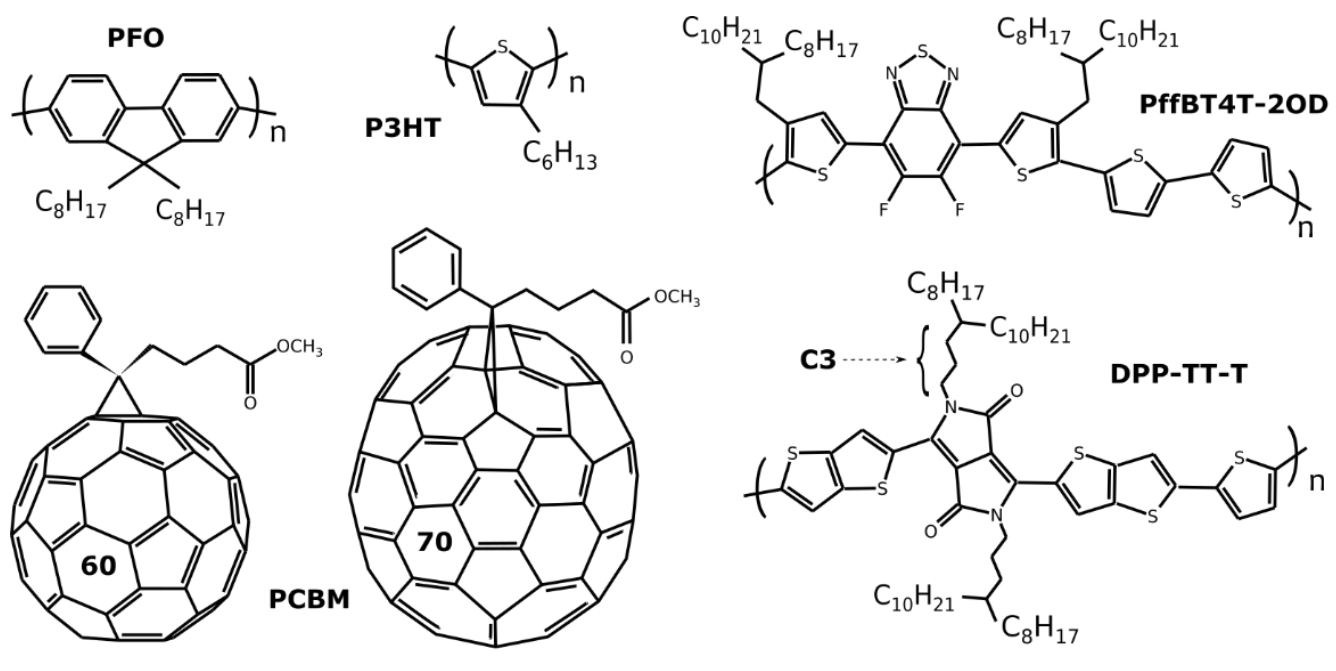

Figure 15.1: Chemical structures of conjugated molecules and polymers considered in this chapter.

In this Chapter, we concentrate on these structure-property relationships, aiming our attention to the UV-VIS spectral range which is relevant for optoelectronic applications. With this focus, we will discuss the dielectric functions of conjugated polymers and how different aspects of their morphology affect them. This will help to explore how the nexus between morphology and optical constants can be exploited to gain information about technologically relevant processes such as blending and monitoring real-time structural evolution during processing. 


\subsection{Basic concepts}

The particular balance of interactions taking place in a conjugated polymer film determines its optical properties. These interactions are strongly affected by morphological variations and therefore there is a strong structure-property nexus. In general, the basic optical behaviour will be given by the properties of the polymer backbone. However, the strong differences observed when comparing solution and solid samples indicate that the optical properties are also modified by the final microstructure resulting from the characteristics of the polymer chains (regio regularity, side chains, weight average molar mass and polydispersity) [7] as well as the processing conditions such as the applied coating technique, the choice of solvent, polymer concentration (or viscosity of the polymer solution), drying conditions, and post-deposition treatments. [810] The interactions with the substrate may also play a role in determining the final structure, as discussed for some polymers in Chap. 5.

\subsubsection{Structure and optical properties}

The structural properties of polymers have been described in Chap. 5 in connection with their physical properties. In the case of conjugated polymers, structural aspects acquire high importance because of their large impact on the electronic structure and concomitantly on their optical properties and suitability for specific applications. The semiconducting character of conjugated monomers and polymers emerges from the bonding via $\mathrm{sp}^{2}$ hybridization among $\mathrm{C}$ atoms.[1] The non-hybridised $\mathrm{p}$ orbitals from neighbouring $\mathrm{C}$ atoms overlap forming $\pi$ bonds, which allow for delocalisation of electrons leading to conducting or semiconducting electronic structures, depending on the bandwidth that results from the number of interacting $\mathrm{C}$ atoms or conjugation length. In common with other organic semiconductors (see Chap. 13), these materials are composed of light atoms and have relatively low packing densities. Consequently, their dielectric constant $\varepsilon_{1}$ takes low values and the dielectric screening of interactions between electrons is limited. Therefore, the Coulomb interaction and the exchange energy between electron pairs with parallel or anti-parallel spin are much stronger than in traditional inorganic semiconductors.[11] One consequence of the low dielectric screening is that photoexcited electron-hole pairs ( $a k a$ excitons) are tightly bound and mostly restricted to molecular sites. [12,13] In addition, intermolecular interactions are usually of the van-der-Waals type, hence weaker than the intra-molecular covalent bonding. As a result, the optical excitations of the solids have strong localised character and resemble the molecular spectra albeit with some shifts and broadenings that depend on the particular microstructure. Several factors contribute to shift and broaden the spectra and it is difficult to disentangle them. 
Considering the polymer as an ensemble of repeating units, the optical spectra will be an inhomogeneously broadened average due to dispersity and different orientations of these polymer molecules. Molecular electronic transitions with vibronic replicas reflect the significant electron-phonon interaction.[14] The frequencies of vibrations that couple to electronic states are usually high, resulting in large total bandwidths that in the polymer frequently give rise to broad electronic transitions. The intensity distribution of the replicas given by the transition probabilities from the 0 -th vibrational level of the ground state to the $\mathrm{m}$-th vibrational level of the excited state, $\mathrm{I}_{0-\mathrm{m}}$, usually corresponds to a Poisson distribution given by $\mathrm{I}_{0-\mathrm{m}}=\left(\mathrm{S}^{\mathrm{m}} / \mathrm{m} !\right) \mathrm{e}^{-\mathrm{S}}$, where $\mathrm{S}$ is the Huang-Rhys parameter that indicates the number of quanta involved in the vibrational excitation. The dominant transition is the $0-0$ (or $\mathrm{S}_{0-1} \leftarrow \mathrm{S}_{0-0}$ ) only when the molecule distortion is small. Variable distances between molecules and conformational disparity are other sources of broadening. These fluctuations have direct influence on the strength of inter- and intramolecular interactions, thus the conjugation length, and with it also the spectral position of the electronic band.[15] In general, an increased conjugation length leads to a spectral redshift. But this spectral signature can correspond to various structural situations.[16] For example, crystalline polymers are actually composed of both crystalline and amorphous regions. In highly crystalline domains the chains form stacks (lamellas) where interactions are favoured and the optical band appears redshifted compared to amorphous domains. Then, the spectral position of the overall band can be correlated with the degree of crystallinity.[17] However, a redshift can also be the result of an increased planarity (larger conjugation length) of the individual chains, especially in the case of short repeat units. In fact, the assignment of spectral signatures observed in a polymer film is quite complex.[18] Many features of the intricate interplay of interactions are captured in the HJ-aggregate model [19] in which the electronically coupled monomers in a single polymer chain behave as a J-aggregate and the $\pi$-stacked chains interact as an $\mathrm{H}$-aggregate. The $\mathrm{J}$ - or $\mathrm{H}$ - character is determined by the sign of the Coulomb coupling which is negative between adjacent repeat units $\left(\mathrm{J}_{\text {intra }}<0\right)$, and positive $\left(\mathrm{J}_{\text {inter }}>0\right)$ between neighbouring chains. However, the overall behaviour of the HJ-aggregate is not intuitive because it depends on the competition between both mechanisms including the particular configuration of the chain and also the disorder.[20] By the mixing of interactions, relative intensities of the vibronic peaks can also deviate from the Poisson distribution. Finally, structural differences can also affect the observed oscillator strength. These can be associated to anisotropic behaviour or to a $3 \mathrm{D}$ isotropic increase in absorption due to conformational changes, especially in increased linearity of chains of rigid monomers.[21]

Table 15.1 summarises expected effects of structural variations on the optical properties of organic semiconductors. Usually, combinations of these effects are present in experimental situations. Particular cases of morphological changes will be presented in Sect. 15.3, focusing in different aspects of conformation and crystallinity. We will also deal with anisotropic optical behaviour in polymeric films. 
Table 15.1: Summary of likely effects on the spectral characteristics of optical spectra expected for several structural situations referred to the case of an isolated monomer or repeat unit.

\begin{tabular}{|c|c|c|c|c|}
\hline Structural change & Cause & Energy shift & Broadening & Optical density \\
\hline Gas to solid & Polarisation & $\downarrow$ & $\uparrow$ & $\approx$ \\
\hline J-aggregation & $\begin{array}{c}\text { Excitonic } \\
\text { coupling }(\mathrm{J}<0)\end{array}$ & $\downarrow$ & $\uparrow$ & $\downarrow$ \\
\hline H-aggregation & $\begin{array}{c}\text { Excitonic } \\
\text { coupling }(\mathrm{J}>0)\end{array}$ & $\uparrow$ & $\uparrow$ & $\approx$ \\
\hline Order & Conjugation & $\downarrow$ & $\uparrow$ & $\uparrow$ \\
\hline Curvature & Persistence & $\approx$ & $\approx$ & \\
\hline
\end{tabular}

\subsubsection{Ellipsometry and dielectric function}

The fundamentals of ellipsometric measurements and analysis have been presented in Chap. 1. Particular considerations and precautions to optimise measurements in samples of conjugated polymers and to extract reliable information from them have been recently reviewed.[6] Usually, spectra acquired at multiple angles of incidence (VASE) and on possibly multiple samples (varying film thickness and or substrate), are considered. Then, to extract material properties from VASE data a multilayer optical model must be built describing the sample(s) under consideration. [22,23] Briefly, the effect of reflection on light polarisation is described applying transfer matrix algebra. An overall reflectance matrix is obtained by multiplying individual matrices that describe reflection at each interface and propagation through each individual layer. Therefore, parameters of the model are, besides the number of layers forming the structure, their thicknesses and dielectric functions. These may be taken from available databases or determined from the studied spectra. The dielectric functions of composite materials as well as roughness and intermixing layers are represented by effective-medium models. With these implementations, the reflectance matrix can be evaluated and the parameters of interest fitted by a least-squares method to the measured spectra.

Two main data analysis approaches are commonly used to determine the optical response of the unknown materials. In a simple case of a film on a known substrate, or when only one of the materials in the multilayer is unknown, it is possible to run a point-by-point numerical fit to obtain $n$ and $k$ by fixing the film thickness to that found from the transparency range imposing $k=0$. Direct point-by-point fits are extremely useful as no assumptions about the spectral dependence of the unknown optical functions are made and arbitrary dispersion relations can be reproduced. An improvement of this numerical procedure is to model $n$ and $k$ by smooth functions such as splines [24] which improves 
the fit convergence and smoothness of the result. The most common approach, however, is to model the optical response of the unknown materials using parameterised analytical functions of the energy according to the physical properties of the studied materials.[23] The principal disadvantage of parametric models is a loss of flexibility in the description of the dielectric function. Only features assumed to be present by the choice of model will be represented in the fit. They do, however, have advantages such as choosing functions that satisfy Kramers-Kronig consistency and can be appropriately correlated to fundamental electronic properties. Moreover, an analytical expression of the optical constants is then available for use in other models and can be parameterised for descriptions of alloys, for instance.

As already discussed, the optical properties of conjugated polymer films can be quite complex due to the basic molecular nature of the polymer and the fact that the particular conformation is decisive to determine the effective optical properties of the film, both in terms of spectral features (reflecting the electronic density of states) and anisotropy (reflecting orientation). Even in a single-component film, a multi-phase morphology is likely to occur. Because of the abundant morphological variations in polymers, this is a case in which a physical parameterisation of the dielectric function that allows describing structural variations and transformations by varying the model parameters is especially useful. As in many other film systems, transparent polymers (or measurements in the transparency region) may be modelled by a Cauchy dispersion,[23] which is useful to investigate film thickness or whether there is optical anisotropy, for instance. However, in the majority of cases it is necessary to represent the absorption bands. Several authors performed systematic studies to compare how well different line-shapes described ellipsometry data for thin conjugated polymer and macrocycle films.[25-27] These studies established that it was essential to allow for asymmetric lineshapes in order to reproduce the experimental measurements. Taking into account the physical nature of the electronic excitations in conjugated polymers, the standard critical point model (SCP) provides the most consistent description and it was proven to fit best the experimental data with the minimum number of parameters. It is based on the expression of the dielectric function due to a critical point $(\mathrm{CP}):[11,28]$

$$
\varepsilon_{\mathrm{CP}}(\omega)=\mathrm{C}-\mathrm{A} \mathrm{e}^{\mathrm{i} \phi}\left(\hbar \omega-\mathrm{E}_{\mathrm{g}}+\mathrm{i} \Gamma\right)^{\mathrm{n}},
$$

where $\mathrm{n}=\mathrm{D} / 2-1$ is related to the dimensionality $\mathrm{D}=3,1,0$ of the $\mathrm{CP}$ (the dependence for $\mathrm{D}=2$ is logarithmic) and the phase angle $\phi$ is a multiple of $\pi / 2$ that identifies its kind (maximum, minimum or saddle point) for each D. In practice, $\phi$ is allowed to take any value as a phenomenological way of accounting for combined shapes which arise from diverse causes such as many-body interactions or even unresolved bands. The amplitude is given by $\mathrm{A}$, the transition energy by $\mathrm{E}_{\mathrm{g}}$, and the lifetime broadening by $\Gamma$. The parameter $\mathrm{C}$ describes a constant background that takes into account contributions in the $\mathrm{UV}$, beyond the measured energy range. The SCP model considers that the dielectric function can be reproduced by a sum of CPs. Strictly, Eq. (15.1) only describes the most resonant term of each $\mathrm{CP}$ contribution to the dielectric function and to improve the fitting of $\mathrm{CP}$ parameters it is advantageous to consider numerical derivatives of the experimentally obtained 
dielectric function. Otherwise, additional broad CPs may be added to account for not constant but weakly varying backgrounds. For example, Arwin et al.[25] performed detailed line-shape analyses of second derivative spectra determined point-by-point on polythiophene thin films. They compared symmetric (Gaussian and Lorentzian) and asymmetric (0D and 3D CPs) models and found that only asymmetric lineshapes were satisfactory to explain the experimental measurements. Other authors compared several models to reproduce the dielectric function itself, confirming this conclusion, for instance on films of several metal phthalocyanines.[26] For many conjugated polymers, [27] the analysis of the ellipsometric data using the SCP model allowed to distinguish between localised excitons for amorphous polymeric films, and 1D/2D delocalisation of the electronic wavefunction for highly crystalline films or containing planar conformations of chains.[27] A description based on a 1D density of states was also employed by Zhokhavets and co-workers in order to estimate the exciton binding energy $\left(E_{b}\right)$ from the dielectric function of polythiophenes.[29] They compared the band edge to a modelled $1 \mathrm{D}$ band gap in order to extract $\mathrm{E}_{\mathrm{b}}$. The obtained $\mathrm{E}_{\mathrm{b}}$ values from this approach were about $0.6 \mathrm{eV}$ for P3OT [29] and also for PFO, [30] in reasonable agreement with exciton binding energies obtained with other techniques [31]. Notice that not always parametric models have a clear physical meaning. For instance, fittings with symmetric lines have been used to investigate morphological issues such as anisotropy [32-34] but typically the oscillators included in such models were not related to the electronic structure of the investigated organic semiconductors.
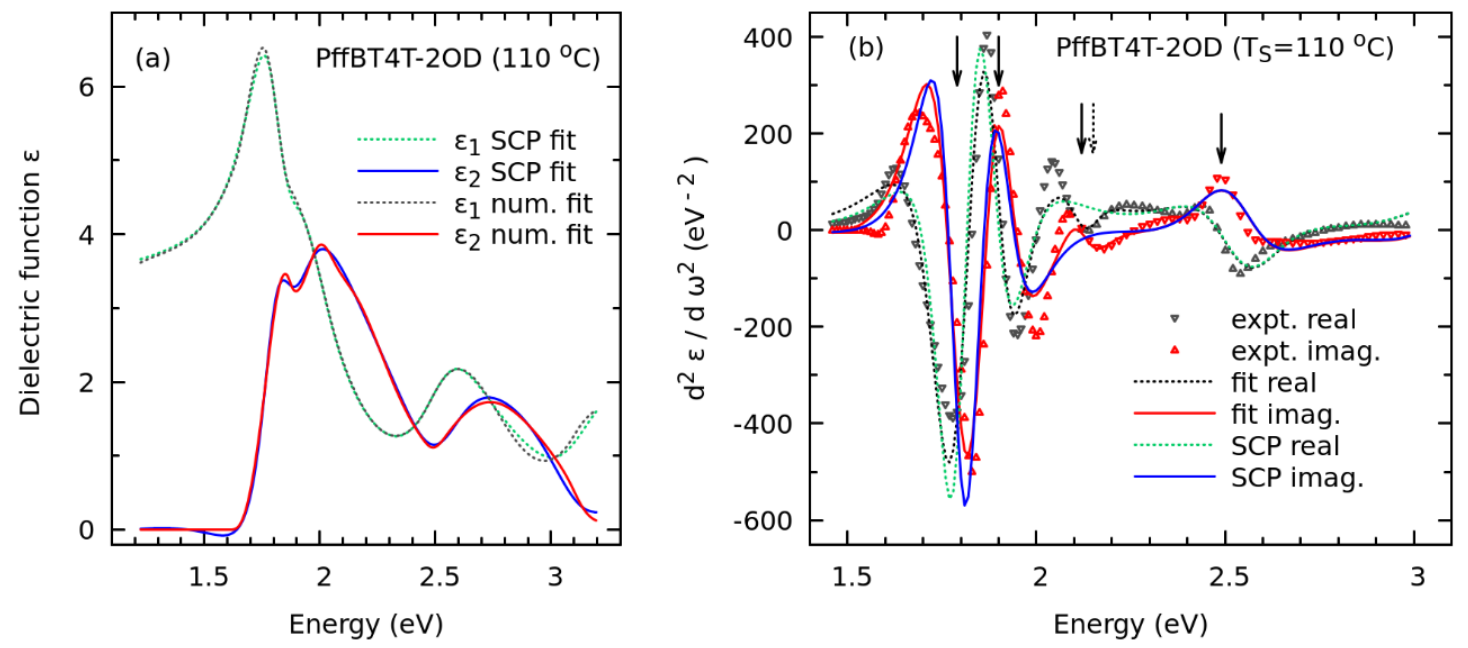

Figure 15.2: (a) Dielectric function of a PffBT4T-2OD film blade coated onto a glass slide at a substrate temperature of $110^{\circ} \mathrm{C}$. The displayed curves compare results obtained both numerically (splines) and parametrically (Standard Critical Point model). (b) Numerically built (expt.) and fitted second derivative spectra compared to the derivative calculated from the parameters of the dielectric function fit using the SCP model. Arrows mark the obtained energies in both ways. The shoulder near $2.1 \mathrm{eV}$ is better resolved by the numerical derivative approach and appears much broader and somewhat shifted for the SCP model.

Figure 15.2 shows an example of dielectric function fitting and lineshape analysis on a poly[(5,6-difluoro-2,1,3-benzothiadiazol-4,7-diyl)-alt-(3,3"'-di(2-ocltyldodecyl)$2,2 ' 5,2$, $; 5 ", 2 "$ "quaterthiopen-5,5"'-diyl)] (PffBT4T-2OD) film deposited at $110{ }^{\circ} \mathrm{C}$. This low band gap polymer is one of the record holding materials with over $11 \%$ 
photovoltaic power conversion efficiency. The parametric SCP model dielectric function reproduces quite well the numerical result. In this case, six 0D CPs were needed to obtain a satisfactory fit of the ellipsometry spectra, see Fig. 15.2 (a), one of them at higher energy and another providing a weakly varying background. The other four CPs (listed in Table 15.2) agree with those present in the numerical second derivative spectra shown in Fig. 15.2 (b) in which the weaker shoulder near $2.1 \mathrm{eV}$ is much better resolved and determined. This comparison illustrates the fact that sharp and isolated transitions can be well determined by fitting the dielectric function but weaker contributions near stronger ones can be distorted by the backgrounds.

Table 15.2. Comparison of the fitted energies and broadenings of the four peaks plotted in Fig. 15.2 (b) using the SCP model dielectric function or the same model applied to the second numerical derivative.

\begin{tabular}{|c|c|c|c|c|}
\hline CP number & 1 & 2 & 3 & 4 \\
\hline $\mathrm{E}_{\mathrm{SCP}}(\mathrm{eV})$ & 1.80 & 1.89 & 2.15 & 2.50 \\
\hline $\mathrm{E}_{2 \text { der }}(\mathrm{eV})$ & 1.79 & 1.90 & 2.12 & 2.49 \\
\hline$\Gamma_{\mathrm{SCP}}(\mathrm{eV})$ & 0.11 & 0.14 & 0.48 & 0.20 \\
\hline$\Gamma_{2 \text { der }}(\mathrm{eV})$ & 0.14 & 0.12 & 0.10 & 0.20 \\
\hline
\end{tabular}

\subsection{Examples of structural variations}

Structural variations occurring in conjugated polymer device-layer films are fundamental to tune and optimise the optical properties for a given optoelectronic application. Enormous efforts have thus been dedicated to understand and control the morphologies that result from different processing routes. At the same time, the correlation between those morphologies and the resulting optical behaviour is of particular interest. In this context, spectroscopic ellipsometry is most helpful to investigate the link between optical properties and film microstructure.

In general, both thermodynamics and kinetics determine the polymer arrangements in the film and complex structures may form with direct impact on the film's spectral optical behaviour. Although different contributions are difficult to disentangle, here we describe three main structural variations that occur in single-phase (one-component) conjugated polymer films and correlate them to observed spectral changes. We distinguish between conformation, crystallinity and anisotropy, which affect in different ways the strengths of the interactions and ultimately the absolute values and/or the energy positions of the optical bands. 


\subsubsection{Conformation}

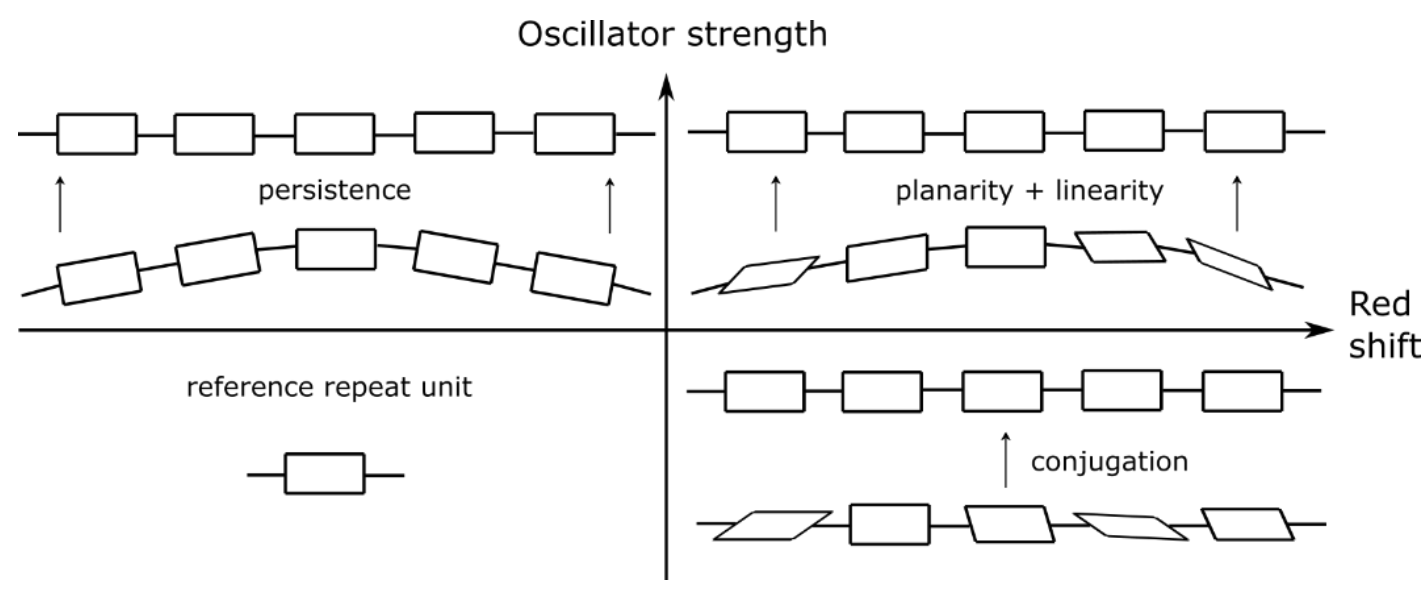

Figure 15.3: Schematic representation of the main conformation variations and their effect on the position and strength of the electronic transitions.

Conformation effects refer to intrinsic molecular properties which result both from steric and electronic interactions. The former derive from the chain regularity, side chain structure, and molecular weight (MW); the latter determine the torsional potentials [35] that regulate the alignment of successive monomers. Various conformation variations and their effect on the optical absorption are schematically depicted in Fig. 15.3. The case of the low-bandgap polymer thieno[3,2-b]thiophene-diketopyrrolopyrrole (DPP-TT-T) is representative and has been well studied. [21] This low-bandgap copolymer shows photostability under prolonged excitation in the low energy absorption band [36] and allows for high field-effect transistor mobilities and good performance as the donor in solar cells. [37] These efficient qualities are correlated to the predominant trans conformation of the polymer chains. In this configuration, the successive monomers are rotated by approximately 180 degrees relative to each other, which leads to more linear oligomer structures than the other limiting conformation case when the successive monomers are equally oriented (cis). In the cis structure, the backbone is curved within the conjugated plane and results in lower optical absorption.[21] Note that in this case more curved or more linear long oligomers differ in the oscillator strength but not, to a first approximation, in the transition energies. The observed effect is associated to the concept of persistence length, $\lambda_{\mathrm{p}}$, which can be thought as a relative propensity of the polymer to adopt a linear structure. On the other hand, the transition energies are closely related to the conjugation length, which structurally is associated to planarity. It is interesting to think how one chain could be linear but not necessarily planar (if adjacent monomers are rotated with respect to each other). Similarly, one chain could be planar but have more or less curvature, if each repeat unit is bonded in cis or trans conformations. In addition, the same effect was observed in solution and was insensitive to dilution, confirming the intrinsically high $\lambda_{p}$ value in DPP-TT-T. [21] Figure 15.4 
shows that this effect is enhanced both with the position of the branching point on the polymer side chains [38] and even more with the molecular weight of the polymer, which favours an extended $\lambda_{\mathrm{p}}$.

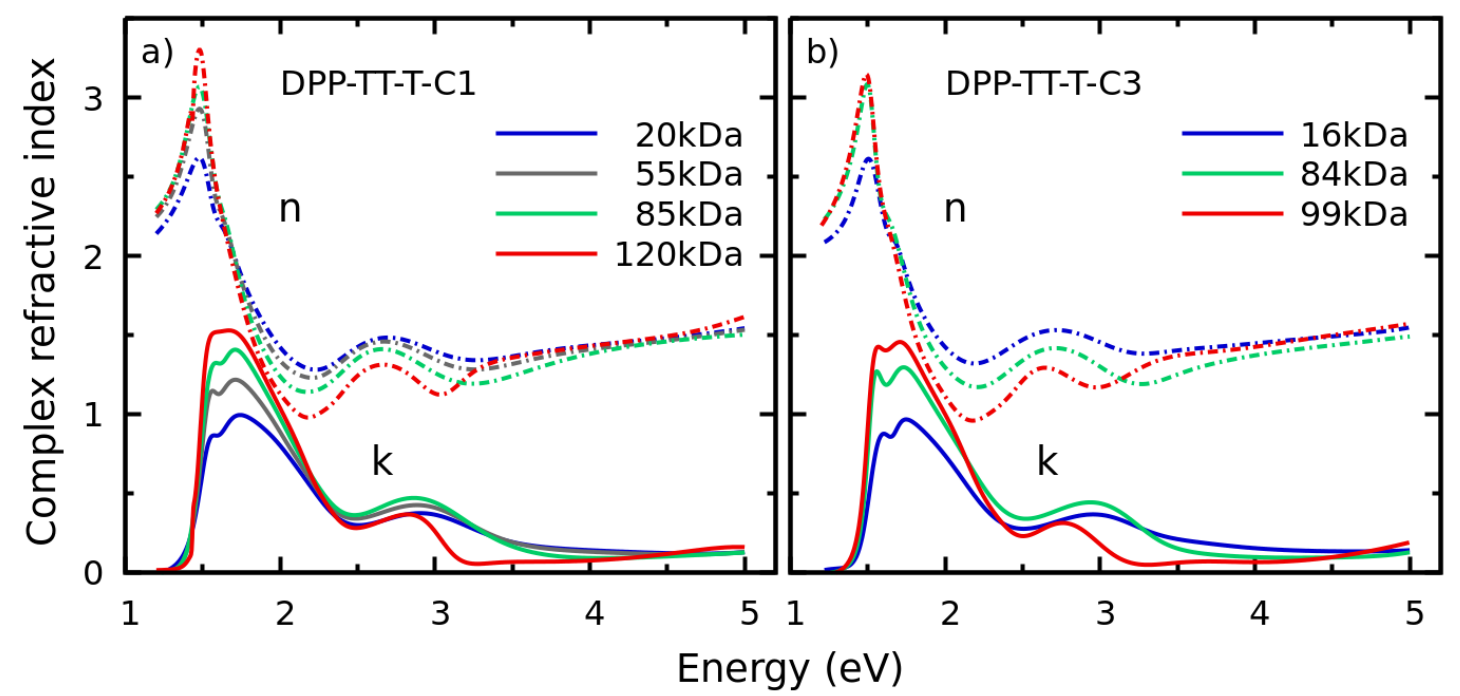

Figure 15.4: Optical constants (n, k) for pristine polymer films of DPP-TT-T with (a) C1 and (b) C3 branching points (the synthesis details are given in Ref.[38]) and for several number-average molecular weights (adapted from Ref. [21]).

A different example of conformation variation is the case of the blue-light emitting conjugated polymer poly(9,9-dioctylfluorene) (PFO).[39] This material appears in a diversity of polymorphic forms [40,41] with different values of torsion angle between monomers. The most interesting conformer is the $\beta$-phase in which the torsion angle between adjacent monomers is $180^{\circ}$. In contrast, most solution processed films contain a broad distribution of torsion angles leading to a wormlike chain conformation or glassy structure. In this respect, $\beta$-phase represents an example in which planarity is enhanced and thus conjugation length is increased. In fact, the optical features associated to this phase are a new transition altogether. Figure 15.5 shows the refractive indices measured for two spin-coated films of PFO. In the glassy $+\beta$ sample a fraction of chain segments was driven to adopt the planar conformation, giving rise to a distinct peak at lower energy even if the $\beta$-phase fraction is below $10 \%$. In this case, compared to the predominant disordered phase, since the fluorene units are relatively short the well-defined planar chain segments have a higher conjugation length, even if no crystallisation takes place. $[41,42]$ 


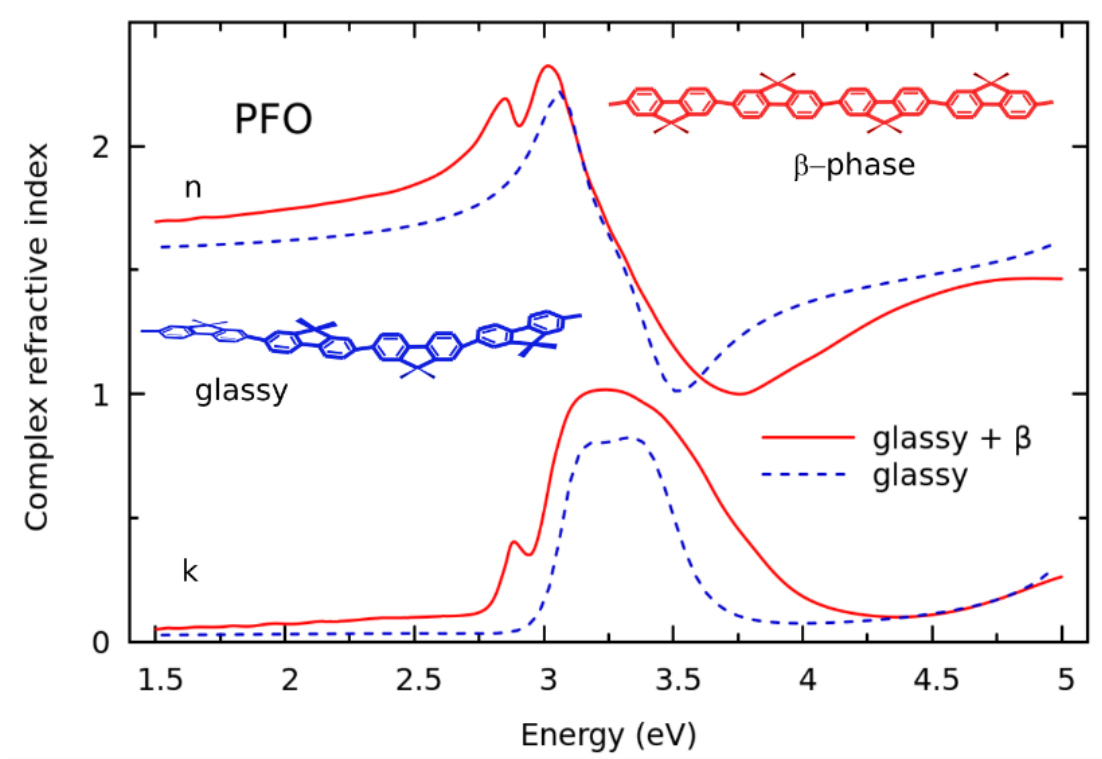

Figure 15.5: Optical constants (n, k) for glassy and glassy $+\beta$ PFO films (see Ref.[27]) as determined by ellipsometry. The low energy absorption feature appears due to the higher conjugation length characteristic of the planar $\beta$-phase conformation (see sketch).

\subsubsection{Crystallinity}

Crystallisation refers to the aggregation of the polymer chains in a regular or ordered fashion. Obviously, the degree of stereoregularity and the strength of intermolecular interactions affect the ability of a polymer to form crystalline domains, which are stacks of orderly folded chains (called lamellas). For a material with tendency to crystallise, the degree of crystallisation will strongly depend on molecular weight, as entanglement between chains appears in long molecules. Kinetic factors also have a large influence in crystallisation processes: solution processing often results in kinetically-trapped out-ofequilibrium microstructures. However, a highly crystalline polymer is rarely entirely ordered, this means that there are amorphous regions between lamellas and the degree of crystallinity (or paracrystallinity) is a parameter by itself. Frequently, lamellas tend to order forming spherical regions from a nucleation point called spherulites, i.e. spherical crystallites. (In thin films, the equivalent 2D structures with circular symmetry are still called spherulites.) Amorphous domains are still present within the spherulites or between their boundaries.

A well-known example of steric effect occurs in poly (3-hexylthiophene) (P3HT) which contains a mixture of regioisomers. The relative ratio of these regioisomers in the resulting polymer gives the regioregularity. Highly regioregular RR-P3HT tends to crystallise easily and is preferable for applications such as transistors and photovoltaics as both, electrical and optical properties are enhanced (see Chap. 12). On the contrary, the irregular substituent distribution in regiorandom RRa-P3HT forces the thiophene units to twist away from planarity, hindering crystallisation.[44] Figure 15.6 compares the 
refractive indices of regiorandom (amorphous) P3HT and two RR-P3HT samples with different degrees of crystallinity. The spectrum of RRa-P3HT is blue shifted and displays smaller oscillator strength compared to the RR counterparts. The former is due to the reduction in conjugation length associated to the frequent twists forced by steric interactions of the side chains. The latter is a combination of a smaller average density (amorphous chains cannot pack as densely as crystalline ones) and a lower degree of linearity in the chain (as it was discussed in section 15.3.1) The dielectric function is proportional to the density of dipoles, and this is in most cases proportional to the material density (see e.g., [39]). Interestingly, the optical properties are also clearly affected by the degree of crystallinity [20,45]. As it was discussed in section 15.2.1, the main optical transitions have vibronic replicas whose intensity is described by the Huang-Rhys parameter. Samples with higher crystallinity often result in higher intensities for the lowest vibrational levels with respect to higher energy vibronic peaks. Moreover, the broadening of each transition and vibronic sideband increases with disorder. To be able to distinguish vibronic sidebands is, in itself, a sign of chain ordering. For instance, $x$-ray diffraction shows that both PffBT4T-2OD (Fig. 15.2) and DPP-TT-T (Fig. 15.4) are partially ordered. Increasing ordering in DPP-TT-T by reducing curvature leads not only to higher overall oscillator strength, but also, to a redistribution of peak intensities in favour of the lowest energy transitions, as observed in Fig. 15.4.

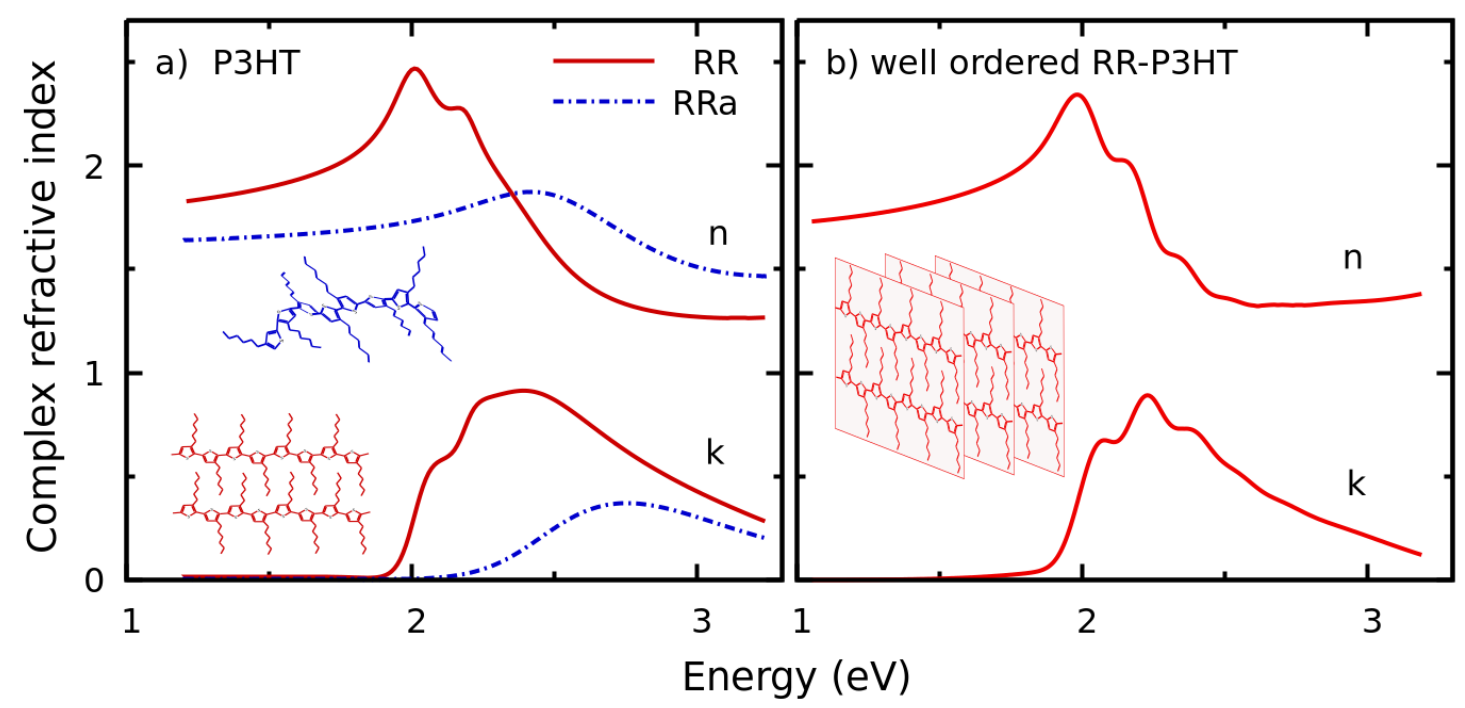

Figure 15.6: Optical constants (n, k) for P3HT with different crystallinity. a) Spectral differences observed in films of amorphous regiorandom (RRa) and device-quality semicrystalline regioregular (RR) material. The sketches illustrate that only the stereoregular chains are able to form crystalline domains. (b) Spectrum of a well-ordered film of crystalline P3HT according to Ref. [43]. The sketch indicates that large crystallites are associated to this spectrum. 


\subsubsection{Anisotropy}

Anisotropic optical properties originate both from the intrinsic anisotropy of polymer chains and from the material microstructure. Since most conjugated polymers behave as rigid-rod-like macromolecules, even amorphous solution-processed polymer films may exhibit preferential molecular orientation (in-plane vs. out-of-plane) giving rise to a uniaxial dielectric tensor. Therefore, evaluation of the optical anisotropy is necessary to correctly establish the oscillator strength of the transitions.[6] Higher degree of orientation of the film associated to an increased alignment of the chains tends to enhance the anisotropy, leading to different values of the tensor components as well as varying transition energy positions. This is similar to the case of films made from ordered organic small molecules (see Chap. 13). Certain mixtures of orientations of ordered as well as disordered phases normally coexist in the films giving an effective anisotropic behaviour which averages to a higher symmetry [46] than that of a perfectly ordered domain.

Figure 15.7 shows the complex refractive indices determined by ellipsometry on a regioregular (>90\%) P3HT film with both random polycrystalline and oriented spherulitic regions. [47] Interestingly, the area where orientation was suppressed was still highly crystalline and its isotropic optical properties showed spectral features typical of crystalline P3HT. An example of a film containing spherulites is shown in the figure. The Maltese cross patterns centred at each spherulite viewed between crossed polarizers evidence their birefringent nature and the radial disposition of polymer chains, which can be parallel or perpendicular to the radial direction. The anisotropic optical response in the measured spherulitic region was well represented, within experimental error, by a uniaxial model with a variable in-plane optic axis aligned with the fibres. In this case, as shown in Fig. 15.7, the index along the fibres is higher than perpendicular to them, indicating that the polymer chains align parallel to the fibres. Although the two reported crystalline polymorphs of regioregular P3HT are monoclinic,[48] domain misalignment and amorphous interlamellar material leads to at most orthorhombic-like dielectric tensor, for example in biaxially strained films.[44] Crystallinity can be approximately evaluated by considering that the spectra can be represented by an average of $\mathrm{RR}$ and $\mathrm{RR}$ a mixtures. [49] For the case of Fig. 15.7, this gives a qualitative estimation of almost complete crystallinity parallel to the fibres whereas the isotropic and the perpendicular spectra contained about $10-20 \%$ of RRa spectral contribution.[47] 


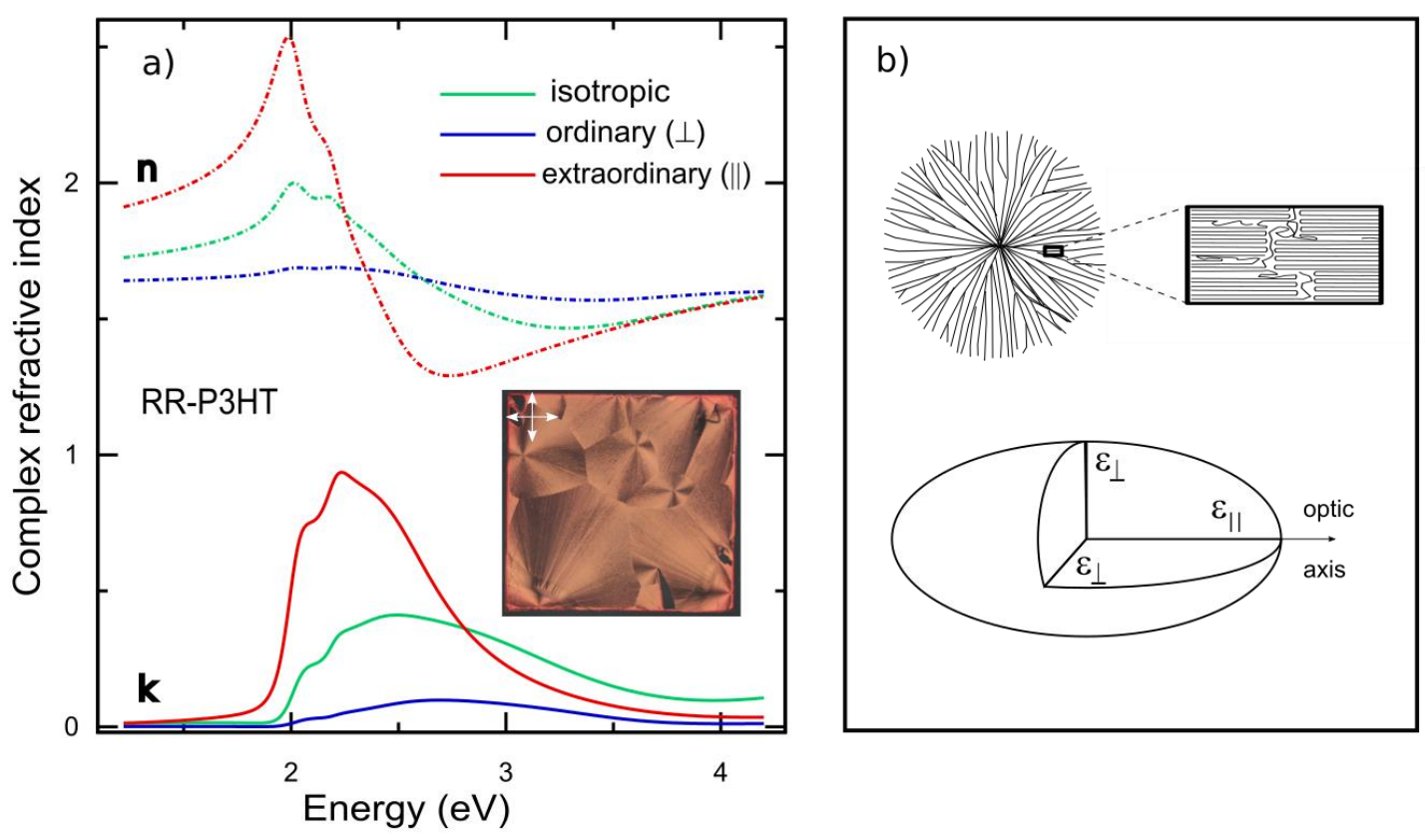

Figure 15.7: a) Complex refractive index (n, $k$ ) data in two zones of a RR-P3HT film. The anisotropic components were measured on a spherulite and the isotropic curves correspond to a region where orientation was suppressed. The inset shows a film $\left(2.5 \times 2.5 \mathrm{~cm}^{2}\right)$ with spherulites viewed between crossed polarizers. Adapted from Ref. [47]. b) Sketch of a spherulite and preferential orientation of the lamellas within the fibrils according to the deduced uniaxial dielectric tensor represented below.

\subsection{Blending}

Mixing different materials into composites is often utilised in the organic semiconductor field. For instance, white lighting can be achieved by blending blue, green and red emitting materials with energy alignment as to produce cascade energy transfer between them. In photovoltaics, blending electron donor and acceptor molecules into the so called bulk heterojunction has emerged as the most effective way of simultaneously obtaining efficient charge separation of the photoinduced excitons while maintaining good light absorption (for which relatively thick films are required). The optical properties of blended films are, in general trends, a combination of the optical properties of the constituents weighted by the corresponding volume fraction. Figure 15.8 shows the complex refractive index of blends of DPP-TT-T with the acceptor PC70BM. The absorption profile includes the transitions of the corresponding components. For instance, the main absorption band of the polymer (Figure 15.4) is also easily observed in the blend. Not just that, the increase in absorption obtained with increasing molecular weight is also preserved in the blends.

The new optical transitions that emerge when mixing, such as the charge transfer excitons, have absorption strengths that are several orders of magnitude smaller than the transitions of the individual components.[50] In this respect, one could think of using effective medium approximations (EMAs, see Chap. 1) to describe the optical properties of these 
blends provided that the two materials are well mixed in comparison to the wavelength of light. In good solar cell systems such as those described in Ch. 12, this is the case as the domain size is limited to a few tenths of nanometers (roughly twice the exciton diffusion length). The major difficulty arises, however, from the fact that during the deposition of the film the two materials interfere in the way each other would solidify when alone. It is known, for instance, that fullerenes tend to partially prevent polymer crystallisation. In terms of the optical properties this would mean that the optical properties of each individual component are not the same as the optical properties of that component when mixed. It has been reported, for instance, that the degree of anisotropy decreases with blending.[51] Moreover, the two components may be inhomogeneously distributed in the vertical direction (perpendicular to the plane of the substrate), forming composition profiles of the two species. [52] This will depend on the surface energies as well as the solubilities of the different compounds in the solvent and additives used, and ellipsometry can be employed to determine such segregation.

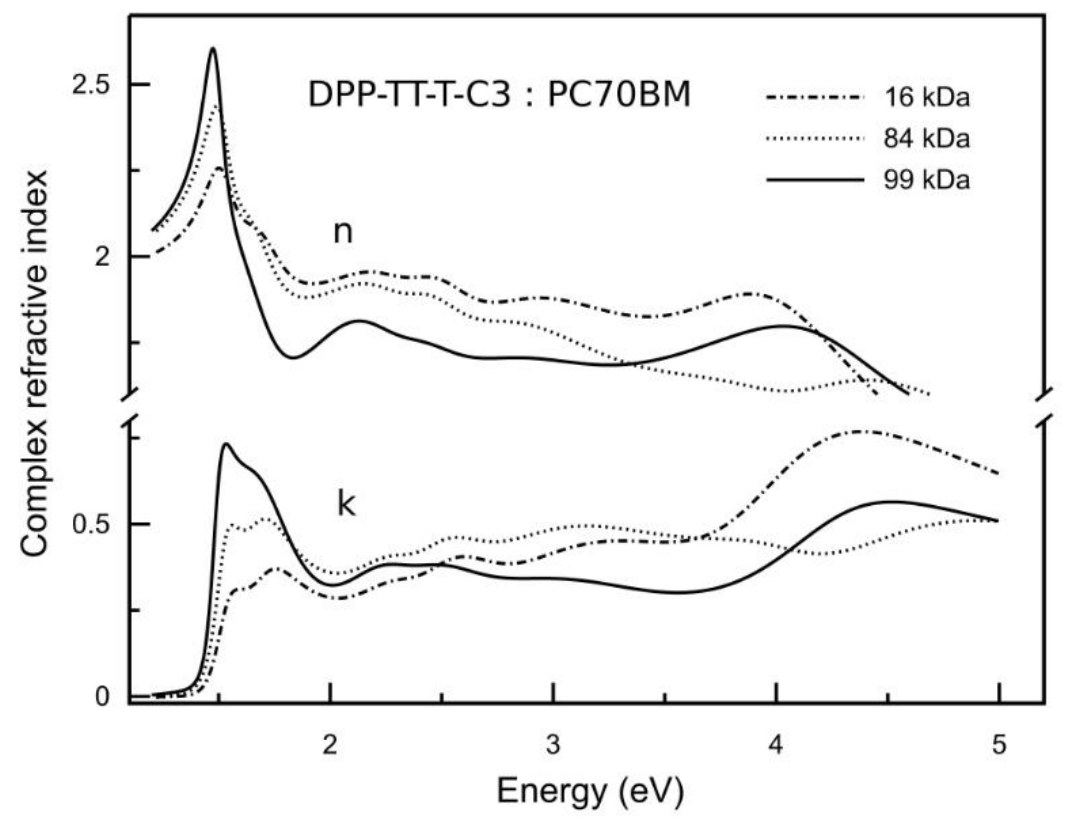

Figure 15.8: Optical constants (n, k) for blend films of DPP-TT-T-C3 and PC70BM for several numberaverage molecular weights of the polymer (cf. Ref.[21]).

Since it is difficult to predict a priori the effect of blending on the morphology and subsequent optical properties, blends are often treated as a completely new material, as though it was an alloy. Otherwise, advanced effective medium approximations are required which include the fact that the degree of crystallinity and orientation can vary. $[53,54]$

When one of the two compounds has a robust morphology, i.e. approximately independent of processing, ellipsometry can be used to evaluate if the optical properties of the other component have changed upon blending. For instance, blends of amorphous 
polythiophene (RRa-P3HT) with two types of fullerenes revealed that for small amounts of fullerene there is mixing at the molecular level, i.e. the amount of small molecules is below the miscibility limit within the polymer matrix.[55] In this context, ellipsometry was used to deduce the optical properties of the fullerene component in the blend, demonstrating the disappearance of the absorption bump often associated to fullerene aggregation.

\subsection{Monitoring in real time}

The strong nexus that exists between the optical and structural properties on conjugated polymer thin films implies that real time ellipsometry can be employed to infer important structural information. Perhaps the simplest case corresponds to following the morphology as a thin film experiences thermally induced phase transitions. In Sect. 15.3.2. we explained that the crystalline and the amorphous versions of a given polymer have substantially different refractive indices. By exposing a thin film to a ramp of temperature, it is possible to actually monitor the different phases directly in a single material film. Figure 15.9. shows the temperature dependent refractive index for regio regular P3HT (after [56]). As the temperature is increased, the film expands reducing like this the density of dipoles and thus the oscillator strength. Thermally activated vibrations statistically decrease the conjugation length, and thus gradual blue shifts are found upon heating. Peaks also broaden with temperature, becoming less well resolved. These general trends can abruptly change when the temperature is raised above a characteristic phase transition temperature. For instance, the speed at which the aforementioned effects happen will accelerate when taking the film above the glass transition temperature. The transition between crystalline and molten film is, perhaps, the one that results in the largest variation in optical properties, as shown in Figure 15.9. Indeed, the optical properties of the molten film resemble those of the regio random P3HT (see Figure 15.6.). It has been shown that the temperature dependence of the ellipsometric raw data can be used to monitor phase transitions. [6] Figure 15.9. b) and c) exemplify this for a RR-P3HT film with a kink observed for the glass transition temperature, while a sudden change is found upon melting (on heating) or crystallisation (on cooling).

Since phase transitions can depend on the geometrical confinement imposed by the thin film geometry, their corresponding temperatures often vary with respect to the bulk. [57,58] The average thermal transition temperature depends thus on film thickness. Recently, ellipsometry has been employed to determine the profile of phase transition temperatures through the depth of thin films. Interestingly, the phase transition temperatures at the free surface have been found to be higher than those at the buried interphase, possibly due to the enhanced polymer packing at the surface granted by the extra free volume. [56] 

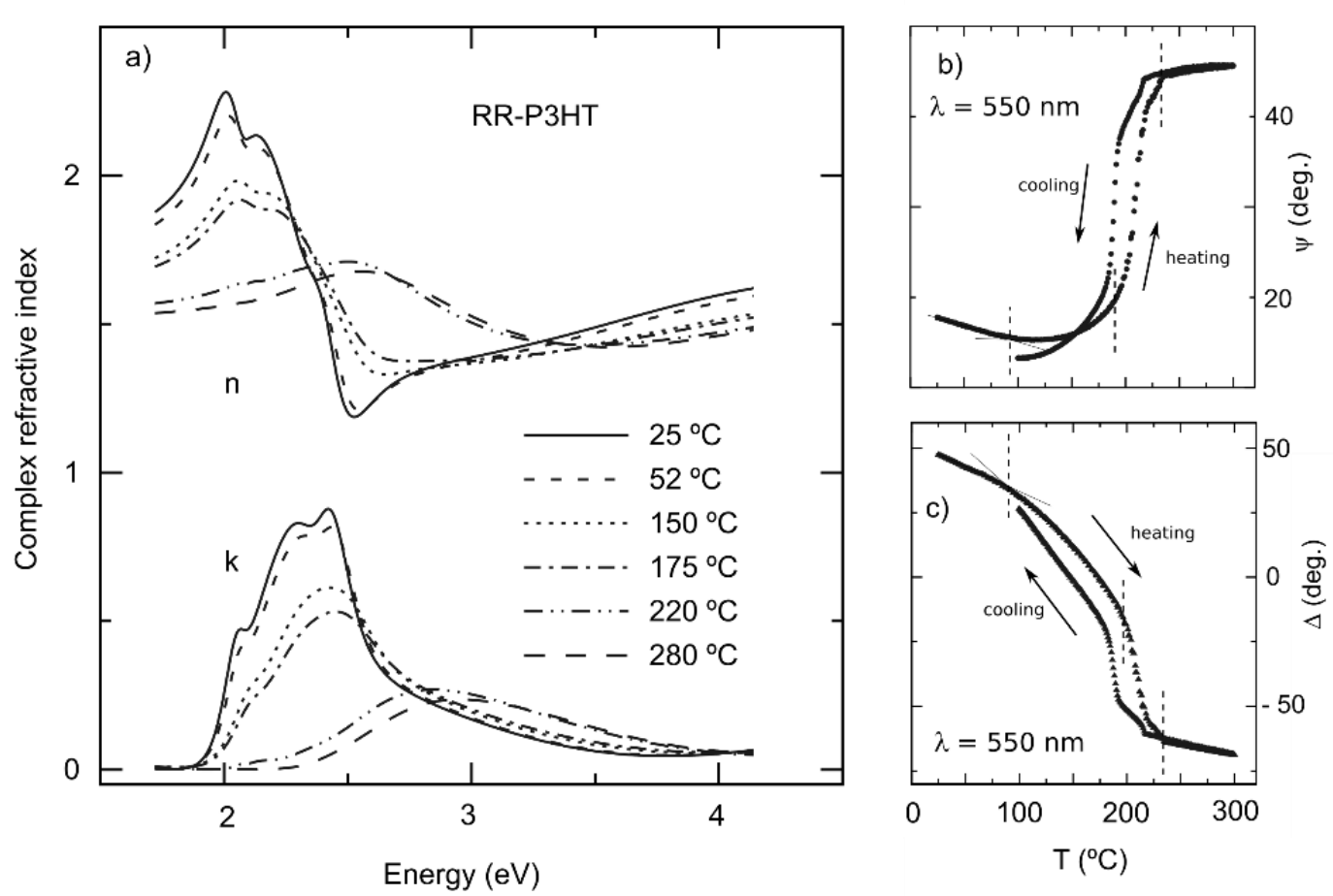

Figure 15.9: (a) Optical properties of regio regular $\mathrm{P} 3 \mathrm{HT}$ as a function of temperature (adapted from reference [56]). The kinks just below $100^{\circ} \mathrm{C}$ observed in the measured ellipsometric angles in (b) and (c) are due to the glass transition, and the larger changes near $200^{\circ} \mathrm{C}$ correspond to melting or crystallisation (see text).

Thermal annealing can also be employed in blend films and monitored upon annealing by means of ellipsometry. Indeed, the phase diagram of polymer and fullerene blends can be determined using this technique.[59] The polymer crystallinity, which can be partially frustrated when processing both materials simultaneously, may be restored by thermal or vapour annealing the films. [52] The crystallisation process is accompanied by molecular diffusion in the out of equilibrium structure formed from quenching the solution, as ordering could not be achieved without expelling foreign molecules from the inside of a forming crystallite. Correlation between these two processes has been followed using ellipsometry for the case of P3HT blended with PCBM.[52] The complexity of blends makes them, however, a very difficult system to model. An interesting way of investigating diffusion processes isolated from crystallisation was proposed by the NIST group.[60] They fabricated bilayer structures of the donor and acceptor types of molecules and ellipsometrically followed the composition of each of the sublayers when applying temperature. They found that the glass transition of the polymer sets the onset for fullerene diffusion. Moreover, they investigated the miscibility limits using this approach.

Film formation has also been investigated using ellipsometry during the deposition itself. The film thinning upon solvent removal can be easily determined spectroscopically. Moreover, the solidification and even the existence of pre-aggregates in solution can be identified with in situ ellipsometry.[9,10] This technique can also be employed to investigate the role of solvent additives during the film deposition. For instance, ellipsometry collected during drying of a solution of the high performing PffBT4T-ODT 
(see Figure 15.1 for chemical structure) blended with PC70BM showed that there are two main drying regimes.[61] First, the wet film thins by evaporating the main carrier solvent, greatly increasing the solid content concentration, and then the solvent additive leaves very slowly the viscous film. During the second regime, the polymer still enhances its crystallisation, as the solvent additive mainly dissolves the fullerene.

These very advanced experiments are paving the way for the use of ellipsometry as an in situ quality monitoring tool during the fabrication of roll to roll photovoltaic modules (see Ch. 12). [62,63]

\subsection{Summary}

In this chapter we have explained the nexus between the ellipsometrically deduced optical properties and the film morphology in polymer semiconductors. We have first given an introduction to the electronic properties of conjugated polymers and how the way in which molecules arrange in the solid state can alter the electronic and optical properties versus isolated polymer chains. We have then correlated these with their dielectric function, describing both the numerical inversion of the ellipsometric data as well as the most common parametric description of the dielectric function. The following sections explained the effect of conformation, crystallinity and molecular orientation on the optical properties. Since blends of different materials are often the technologically relevant architecture, especially in organic photovoltaics through the bulk heterojunction concept, we devoted some attention to the optical properties of blend films focussing on the deviations observed experimentally from the basic mixing theories. In the final section we reviewed some of the latest results on the use of in situ ellipsometry in polymer semiconductors, and how the optical properties can be used as a proxy to address the major morphological changes happening upon phase transition or during film formation.

\section{Acknowledgments}

The authors acknowledge financial support from the Spanish Ministry of Economy and Competitiveness through grant MAT2015-70850-P and the Severo Ochoa Programme for Centres of Excellence in R\&D (SEV-2015-0496).

\section{References}

[1] A. Köhler and H. Bässler, Electronic Processes in Organic Semiconductors.: Wiley-VCH Verlag, Weinheim, (2015).

[2] S. Günes, H. Neugebauer, and N. S. Sariciftci, Chem. Rev. 107, 1324-1338 (2007).

[3] J. H. Burroughes, D. D. C. Bradley, A. R. Brown, R. N. Marks, K. Mackay, R. H. Friend, P. L. Burn, and A. B. Holmes, Nature 347, 539-541 (1990). 
[4] K.-J. Baeg, M. Caironi, and Y.-Y. Noh, Adv. Mater. 25, 4210-4244 (2013).

[5] J. Liu, Y. Shi, and Y. Yang, Adv. Funct. Mater. 11, 420-424 (2001).

[6] M. Campoy-Quiles, M. I. Alonso, D. D. C. Bradley, and L. J. Richter, Adv. Funct. Mater. 24, 21162134 (2014).

[7] K. Koynov, A. Bahtiar, T. Ahn, R. M. Cordeiro, H.-H. Horhold, and C. Bubeck, Macromol. 39, 8692-8698 (2006).

[8] U. Zhokhavets, G. Gobsch, H. Hoppe, and N. S. Sariciftci, Thin Solid Films 451, 69-73 (2004).

[9] M. Campoy-Quiles, M. Schmidt, D. Nassyrov, O. Peña, A. R. Goñi, M. I. Alonso, and M. Garriga, Thin Solid Films 519, 2678-2681 (2011).

[10] T. Wang, A. D. F. Dunbar, P. A. Staniec, A. J. Pearson, P. E. Hopkinson, J. E. MacDonald, S. Lilliu, C. Pizzey, N. J. Terrill, A. M. Donald, A. J. Ryan, R. A. L. Jones, and D. G. Lidzey, Soft Matter 6, 4128-4134 (2010).

[11] P. Yu and M. Cardona, Fundamentals of Semiconductors: Physics and Materials Properties.: Springer-Verlag, Berlin, (2010).

[12] U. Rauscher, H. Bässler, D. D. C. Bradley, and M. Hennecke, Phys. Rev. B 42, 9830-9836 (1990).

[13] S. Heun, R. F. Mahrt, A. Greiner, U. Lemmer, H. Bassler, D. A. Halliday, D. D. C. Bradley, P. L. Burn, and A. B. Holmes, J. Phys. Condens. Matter 5, 247-260 (1993).

[14] J. Cornil, D. Beljonne, Z. Shuia, T.W. Hagler, I. Campbell, D.D.C. Bradley, J.L. Brédas, C.W. Spangler, and K. Müllen, Chem. Phys. Lett. 247, 425-432 (1995).

[15] K Pichler, D A Halliday, D D C Bradley, P L Burn, R H Friend, and A B Holmes, J. Phys.: Condens. Matter 5, 7155 (1993).

[16] S. Giri, C. H. Moore, J. T. Mcleskey, and P. Jena, J. Phys. Chem. C 118, 13444-13450 (2014).

[17] M. Campoy-Quiles, V. Randon, M. Mróz, M. Jarzaguet, M. Garriga, and J. Cabanillas-González, Org. Photon. Photovolt. 1, 11-23 (2013).

[18] D. Raithel, S. Baderschneider, T. B. de Queiroz, R. Lohwasser, J. Köhler, M. Thelakkat, S. Kümmel, and R. Hildner, Macromol. 49, 9553-9560 (2016).

[19] F. C. Spano and C. Silva, Annu. Rev. Phys. Chem. 65, 477-500 (2014).

[20] F. C. Spano, J. Chem. Phys. 122, 234701 (2005).

[21] M. S. Vezie, S. Few, I. Meager, G. Pieridou, B. Dörling, R. S. Ashraf, A. R. Goñi, H. Bronstein, I. McCulloch, S. C. Hayes, M. Campoy-Quiles, and J. Nelson, Nat. Mater. 15, 746-753 (2016).

[22] R. M. A. Azzam and N. M. Bashara, Ellipsometry and Polarized Light.: North-Holland, Amsterdam, (1977). 
[23] H. G. Tompkins and E. A. Irene, Handbook of Ellipsometry.: William Andrew Publishing, Springer, Heidelberg, (2005).

[24] M. Garriga, M. I. Alonso, and C. Domínguez, Phys. Stat. Sol. B 215, 247-251 (1999).

[25] H. Arwin and R. Jansson, Electrochim. Acta 39, 211-215 (1994).

[26] Z. T. Liu, H. S. Kwok, and A. B. Djurisic, J. Phys. D: Appl. Phys. 37, 678 (2004).

[27] M. Campoy-Quiles, J. Nelson, D. D. C. Bradley, and P. G. Etchegoin, Phys. Rev. B 76, 235206 (2007).

[28] D. E. Aspnes, in Handbook on Semiconductors, T.S.Moss M.Balkanski, Ed.: North-Holland, Amsterdam, vol. 2, ch. 4A, pp. 110-154 (1980).

[29] U. Zhokhavets, R. Goldhahn, G. Gobsch, and W. Schliefke, Synth. Met. 138, 491-495 (2003).

[30] M. Campoy-Quiles, Ph.D Thesis, Imperial College of London (2005).

[31] S. F. Alvarado, P. F. Seidler, D. G. Lidzey, and D. D. C. Bradley, Phys. Rev. Lett. 81, 1082-1085 (1998).

[32] M. Tammer and A.P. Monkman, Adv. Mater. 14, 210-212 (2002).

[33] C.M. Ramsdale and N.C. Greenham, Adv. Mater. 14, 212-215 (2002).

[34] M. Losurdo, M. M. Giangregorio, P. Capezzuto, G. Bruno, F. Babudri, D. Colangiuli, G. M. Farinola, and F. Naso, Macromol. 36, $4492-4497$ (2003).

[35] C. Sutton, T. Körzdörfer, M. T. Gray, M. Brunsfeld, R. M. Parrish, C. D. Sherrill, J. S. Sears, and J.L. Brédas, J. Chem. Phys. 140, 054310 (2014).

[36] S. Wood, J. Wade, M. Shahid, E. Collado-Fregoso, D. D. C. Bradley, J. R. Durrant, M. Heeney, and J.-S. Kim, Energy Environ. Sci. 8, 3222-3232 (2015).

[37] H Bronstein, Z. Chen, R. S. Ashraf, W. Zhang, J. Du, J. R. Durrant, P. S. Tuladhar, K. Song, S. E. Watkins, Y. Geerts, M. M. Wienk, R. A. J. Janssen, T. Anthopoulos, H. Sirringhaus, M. Heeney, and I. McCulloch, J. Am. Chem. Soc. 133, 3272-3275 (2011).

[38] I. Meager, R. S. Ashraf, S. Mollinger, B. C. Schroeder, H. Bronstein, D. Beatrup, M. S. Vezie, T. Kirchartz, A. Salleo, J. Nelson, and I. McCulloch, J. Am. Chem. Soc. 135, 11537-11540 (2013).

[39] M. Campoy-Quiles, G. Heliotis, R. D. Xia, M. Ariu, M. Pintani, P. Etchegoin, and D. D. C. Bradley, Adv. Funct. Mater. 15, 925-933 (2005).

[40] W. Chunwaschirasiri, B. Tanto, D. L. Huber, and M. J. Winokur, Phys. Rev. Lett. 94, 107402 (2005).

[41] A. Perevedentsev, N. Chander, J.-S. Kim, and D. D. C. Bradley, J. Pol. Sci. Part B. Pol. Phys. 54, 1995-2006 (2016).

[42] S. H. Chen, A. C. Su, C. H. Su, and S. A. Chen, Macromol. 38, 379-385 (2005). 
[43] A. J. Morfa, T. M. Barnes, A. J. Ferguson, D. H. Levi, G. Rumbles, K. L. Rowlen, and J. van de Lagemaat, J. Pol. Sci. B: Pol. Phys. 49, 186-194 (2011).

[44] M. C. Gurau, D. M. Delongchamp, B. M. Vogel, E. K. Lin, D. A. Fischer, S. Sambasivan, and L. J. Richter, Langmuir 23, 834-842 (2007).

[45] J. Clark, J.-F. Chang, F. C. Spano, R. H. Friend, and C. Silva, Appl. Phys. Lett. 94, 163306 (2009).

[46] M.I. Alonso and M. Garriga, Thin Solid Films 455-456, 124-131 (2004).

[47] B. Dörling, A. Sánchez-Díaz, O. Arteaga, A. Veciana, M. I. Alonso, and M. Campoy-Quiles, Adv. Opt. Mater. 1700276 (2017). https://doi.org/10.1002/adom.201700276

[48] M. Brinkmann, J. Pol. Sci. Part B: Pol. Phys. 49, 1218-1233 (2011).

[49] D. M. DeLongchamp, R. J. Kline, E. K. Lin, D. A. Fischer, L. J. Richter, L. A. Lucas, M. Heeney, I. McCulloch, and J. E. Northrup, Adv. Mater. 19, 833 (2007).

[50] K. Vandewal, K. Tvingstedt, A. Gadisa, O. Ingänas, and J. V. Manca, Nat. Mater. 8, 904-909 (2009).

[51] M. Campoy-Quiles, C. Müller, M. Garriga, E. Wang, O. Inganäs, and M. I. Alonso, Thin Solid Films 571, Part 3, 371-376 (2014).

[52] M. Campoy-Quiles, T. Ferenczi, T. Agostinelli, P. G. Etchegoin, Y. Kim, T. D. Anthopoulos, P. N. Stavrinou, D. D. C. Bradley, and J. Nelson, Nat. Mater. 7, 158-164 (2008).

[53] S. Engmann, V. Turkovic, P. Denner, H. Hoppe, and G. Gobsch, J. Pol. Sci. B: Pol. Phys. 50, $1363-$ 1373 (2012).

[54] S. Engmann, V. Turkovic, G. Gobsch, and H. Hoppe, Adv. Ener. Mater. 1, $684-689$ (2011).

[55] A. A. Y. Guilbert, M. Schmidt, A. Bruno, J. Yao, S. King, S. M. Tuladhar, T. Kirchartz, M. I. Alonso, A. R. Goñi, N. Stingelin, S. A. Haque, M. Campoy-Quiles, and J. Nelson, Adv. Funct. Mater. 24, 6972-6980 (2014).

[56] C. Müller, L. M. Andersson, O. Peña-Rodriguez, M. Garriga, O. Inganäs, and M. Campoy-Quiles, Macromol. 46, 7325-7331 (2013).

[57] M. Campoy-Quiles, M. Sims, P. G. Etchegoin, and D. D. C. Bradley, Macromol. 39, 7673-7680 (2006).

[58] A. Roigé, M. Campoy-Quiles, J. O. Ossó, M. I. Alonso, L. F. Vega, and M. Garriga, Synth. Met. 161, 2570-2574 (2012).

[59] C. Müller, J. Bergqvist, K. Vandewal, K. Tvingstedt, A. S. Anselmo, R. Magnusson, M. I. Alonso, E. Moons, H. Arwin, M. Campoy-Quiles, and O. Inganäs, J. Mater. Chem. 21, 10676-10684 (2011).

[60] D. Leman, M. A. Kelly, S. Ness, S. Engmann, A. Herzing, C. Snyder, H. W. Ro, R. J. Kline, D. M. DeLongchamp, and L. J. Richter, Macromol. 48, 383-392 (2015). 
[61] H. W. Ro, J. M. Downing, S. Engmann, A. A. Herzing, D. M. DeLongchamp, L. J. Richter, S. Mukherjee, H. Ade, M. Abdelsamie, L. K. Jagadamma, A. Amassian, Y. Liu, and H. Yan, Energy Environ. Sci. 9, 2835-2846 (2016).

[62] S. Logothetidis, Method for in-line determination of film thickness and quality during printing processes for the production of organic electronics, (2014), US Patent App. 14/113,125.

[63] M. V. Madsen, K. O. Sylvester-Hvid, B. Dastmalchi, K. Hingerl, K. Norrman, T. Tromholt, M. Manceau, D. Angmo, and F. C. Krebs, J. Phys.Chem. C 115, 10817-10822 (2011). 